\title{
The temporalis muscle flap for reconstruction of soft palate and lateral oropharyngeal wall after transoral robotic surgery
}

\author{
Giuseppe Meccariello ${ }^{a}$, Filippo Montevecchi ${ }^{\mathrm{a}, *}$, Alberto Deganello $^{\mathrm{b}}$, \\ Giovanni D'Agostino ${ }^{a}$, Chiara Bellini ${ }^{\mathrm{a}}$, Ermelinda Zeccardo ${ }^{\mathrm{a}}$, Claudio Vicini ${ }^{\mathrm{a}}$ \\ ${ }^{a}$ Department of Head-Neck Surgery, Otolaryngology, Head-Neck and Oral Surgery Unit, Morgagni Pierantoni Hospital, Azienda USL della \\ Romagna, Forlì, Italy \\ ${ }^{\mathrm{b}}$ Department of Surgery and Translational Medicine, University of Florence, largo Brambilla 10, 50134, Florence, Italy
}

\section{A R T I C L E I N F O}

\section{Article history:}

Received 5 October 2016

Accepted 28 November 2016

Available online $\mathrm{xxx}$

\section{Keywords:}

Transoral robotic surgery

Reconstruction

Cancer

Palate

Flap

\begin{abstract}
A B S T R A C T
Trans Oral Robotic Surgery (TORS) is a prominent surgical approach for the resection of oropharyngeal tumors without division of the lip and mandible. Some defects following TORS are large and complex enough to benefit soft-tissue coverage. The pedicled temporalis muscle flap is a versatile and reliable flap and may be a valid option to reconstruct defects of the lateral pharyngeal wall and partial soft palate.
\end{abstract}

(c) 2016 Elsevier Ireland Ltd. All rights reserved.

\section{Introduction}

Trans Oral Robotic Surgery (TORS) has emerged as a technique that allows head and neck surgeons to safely resect even large oropharyngeal tumors without dividing the lip and/or the mandible. The resulting defect is usually left to heal by secondary intention; however some defects are large and complex enough to benefit soft-tissue coverage. To enhance safe healing minimizing complications, TORS with reconstruction (whether using free flaps, local flaps, or primary closure) appears to be a superior approach in selected cases, and holds the promise of expanding indications for minimally invasive reconstructive procedures [1]. However, surgical defects following TORS provide a reconstructive challenge because the physical access and visualization of the surgical

\footnotetext{
* Corresponding author. at: Department of Head-Neck Surgery, Otolaryngology, Head-Neck and Oral Surgery Unit, Morgagni Pierantoni Hospital, Azienda USL della Romagna, Viale Forlanini 34, 47100 Forlì, Italy. Fax: +390543735660 .

E-mail address: filippomontevecchi72@gmail.com (F. Montevecchi).
}

field is severely restricted, making the contouring and insertion of the reconstructive tissue more difficult. Here, we report our reconstructive solution using the temporalis muscle flap (TMP) following a TORS resection of a squamous cell carcinoma (SCC) of the of the lateral oropharyngeal wall extending to the soft palate.

\section{Technical description}

A 65 year-old male patient with a cT2N1 SCC p16-, involving the left anterior pillar extending to the soft palate at the left side (Fig. 1A), was referred to our Institution. The history revealed smoking and light drinking habits, no significant comorbidities. The Magnetic Resonance Imaging confirmed a superficial lesion with partial involvement of the soft palate, with a suspicious lymph node at level II. The patient was scheduled for TORS and Selective Neck Dissection (SND) of levels I-IV.

A tracheostomy was performed before proceeding the robotic surgery. Then, a Feyh-Kastenbauer retractor (Gyrus Medical Inc., Maple Grove, MN) was used to expose the 

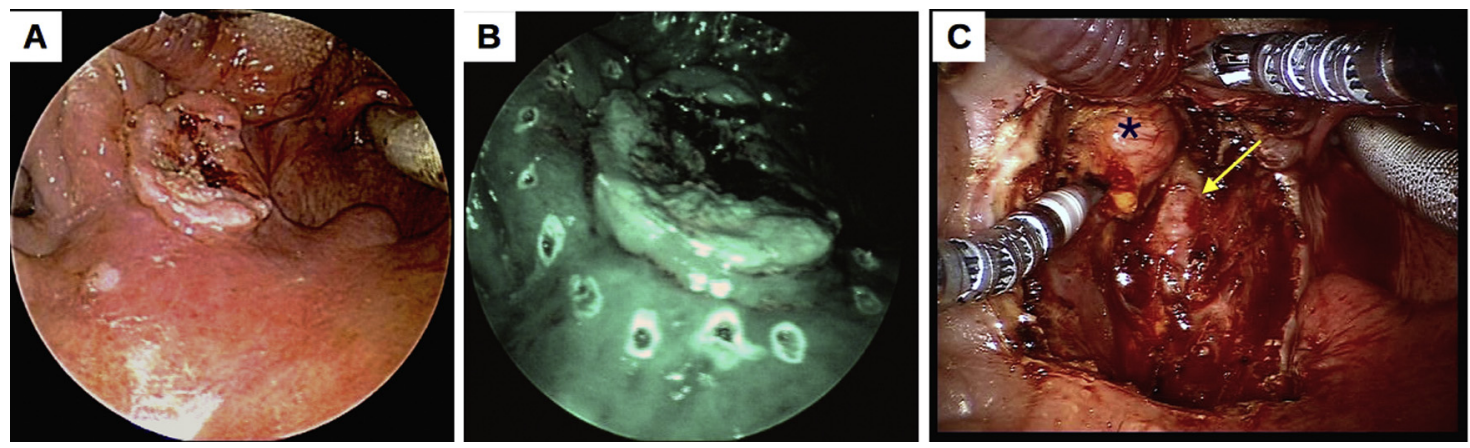

Fig. 1. (A) Endoscopic view of the tumor. (B) Checking of the surgical edges with NBI. (C) Endoscopic view of surgical field after completed tumor resection.
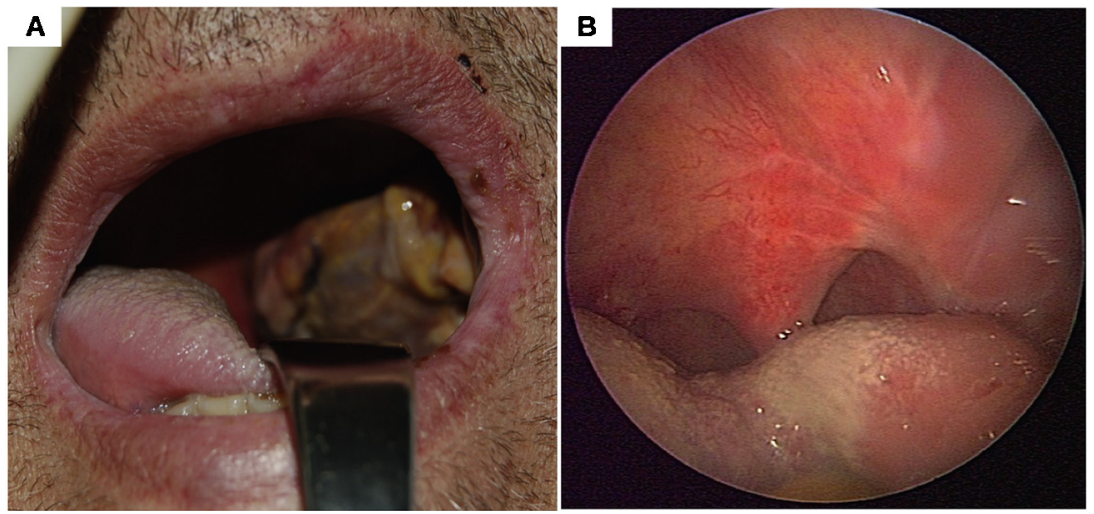

Fig. 2. (A) The temporalis muscle flap on the fifth postoperative day. (B) Endoscopic view of the left lateral pharyngeal and soft palate reconstructed with temporalis muscle flap after one month.

operative field. The tumor margins were observed intraoperatively with a 0 or $30^{\circ} 8 \mathrm{~mm}$ Hopkins Scopes (Karl Storz, Germany) using white light then with the Narrow Band Imaging (NBI) high-definition video-endoscopy system (CV260SL processor, CVL-260SL light source, Olympus Optical Co., Ltd., Japan). The edges of surgical excision were marked with monopolar cautery and controlled with NBI [2] (Fig. 1B). The daVinci ${ }^{\circledR}$ Surgical Robotic System (Intuitive Surgical, Sunnyvale, CA) was positioned $30^{\circ}$ angled on the right side of the patient. A 0 or $30^{\circ} 8.5 \mathrm{~mm}$ endoscopes were used with two 5-mm side arms Maryland dissectors and cautery (Fig. 1C). All vessels encountered during the resection were clipped prior to transaction. The whole surgical specimen was oriented and then submitted to the pathologist for intraoperative assessment of the margin status with frozen sections. Then the SND was performed. Once neck dissection was completed and clear margins confirmed by the pathologist, the TMP was easily harvested [3] and transposed to resurface the defect (Fig. 2). A nasogastric tube was placed. Tracheotomy was closed on postoperative day 5 and the patient resumed oral feeding on day 7 and discharged on day 9 with normal diet. The pathological report was consistent with a pT2N0 R0 SCC p16-. No indications for adjuvant treatments were posed at multidisciplinary tumor board.

\section{Discussion}

Although the current practice following TORS is to allow the defect to heal by secondary intention, many defects are large and complex enough to benefit soft-tissue coverage. In fact, when the carotid sheath is exposed by a communication between the oropharynx and the neck, when a velopharyngeal compromise is anticipated, and when a considerable resection of tongue base tissue has occurred, the transposition of vascularized tissue seems to be beneficial for anatomical restoration and appropriate long-term function. For these situations, the transoral inset of a free flap or of a pedicled flap using robotic surgical visualization and precision would be an appealing reconstructive strategy [4]. In such cases, accurate suture placement for flap insetting in a confined working space remains difficult even using the robot. Therefore, a highly pliable flap, easy to harvest and to transpose, might be a valid option to minimize these issues.

In our case, the transposition of the TMF restored a competent velopharyngeal sphincter and a watertight seal between the pharynx and neck, resulting in timely healing with enhanced postoperative functional results. The TMP is a versatile and reliable flap that is often overlooked in head and neck reconstructive surgery, nevertheless it appears most useful for defects in which the ideal flap is a flexible, tailored muscle with moderate thickness, resulting particularly suitable for upper defects, i.e. following radical tonsillectomy and soft palate resections $[3,5]$. These characteristics fit well in the TORS framework. The minimally invasive surgery requires, whenever possible, a reconstructive approach of the same entity in order to respect the peculiarities of this type of surgery. To the best of our knowledge, this is the first report describing the reconstructive use of TMP after TORS. In terms of 
cost-effectiveness, the use of alternative pedicled flaps in TORS framework probably reduced the risks of postoperative complications, with consequent expenditure restraints. The use of microvascular techniques for these patients might have led to an increase in production costs linked to the greater complexity of the procedure in itself with increase of treatment costs arising from operating room duration and double surgical team [5].

In conclusion, the TMP in TORS might represent a valid option rather than free flaps, especially in elderly patients and patients with significant comorbidities or vessel depleted neck.

\section{Disclosure statement}

Claudio Vicini and Filippo Montevecchi are consultants and proctors for Intuitive Surgical Inc.

\section{References}

[1] Welkoborsky HJ, Deichmüller C, Bauer L, Hinni ML. Reconstruction of large pharyngeal defects with microvascular free flaps and myocutaneous pedicled flaps. Curr Opin Otolaryngol Head Neck Surg 2013;21: 318-27.

[2] Vicini C, Montevecchi F, D'Agostino G, Vito DEA, Meccariello G. A novel approach emphasising intra-operative superficial margin enhancement of head-neck tumours with narrow-band imaging in transoral robotic surgery. Acta Otorhinolaryngol Ital 2015;35:157-61.

[3] Hanasono MM, Utley DS, Goode RL. The temporalis muscle flap for reconstruction after head and neck oncologic surgery. Laryngoscope 2001;111:1719-25.

[4] de Almeida JR, Park RCW, Genden E. M. Reconstruction of transoral robotic surgery defects: principles and techniques. J Reconstr Microsurg 2012;28:465-72.

[5] Deganello A, Gitti G, Parrinello G, Muratori E, Larotonda G, Gallo O. Cost-analysis in oral cavity and oropharyngeal reconstructions with microvascular and pedicled flaps. Acta Otorhinolaryngol Ital 2013;33: $380-7$. 